MedienPädagogik

www. medienpaed.com
Zeitschrift für

Theorie und Praxis

der Medienbildung

ISSN 1424-3636

Themenheft Nr. 15/16: Computerspiele und Videogames

in formellen und informellen Bildungskontexten

\title{
Computerspielen als medial-kulturelle Praktik Ein medienpädagogisch-kulturtheoretischer Zugang zum Phänomen Computerspielen
}

Sabrina Schrammel und Konstantin Mitgutsch

\begin{abstract}
Gegenwärtig werden im medienpädagogischen Diskurs Computerspiele primär als Medienangebote thematisiert, wobei oftmals ausser Acht gelassen wird, dass Spielen eine kulturell geprägte, aktive und soziale Auseinandersetzung mit dem Spiel als Artefakt, Regelwerk und Geschichte impliziert. Im vorliegenden Beitrag wird vor dem Hintergrund bildungstheoretischer und lerntheoretischer Überlegungen ein methodisch-theoretischer Zugang entfaltet und präzisiert, der es erlaubt, Computerspiele als spezifisch situierte medial-kulturelle Praktik pädagogisch zu erfassen, zu beschreiben und zu analysieren. Werden Computerspiele und ihr Spielen als Transformation und Produktion von kulturellen Erfahrungen thematisiert, dann stellt sich die Frage nach der pädagogischen Dimension des Computerspiel(en)s neu. Es eröffnet sich dabei ein Forschungsfeld, das nach differenzierter medienpädagogischer Herangehensweise verlangt. Der vorliegende medienpädagogisch-kulturtheoretische Zugang zum Phänomen Computerspielen baut auf den theoretischen Wurzeln der Spieleforschung auf und fasst das Spielen als medial-kulturelle Handlung.
\end{abstract}

\section{Verortung im Computerspieldiskurs}

Mit der zunehmenden Popularität von Computerspielen geht im wissenschaftlichen Bereich eine vermehrte Auseinandersetzung mit dem Phänomen des digitalen Spielens einher, wobei sich im angelsächsischen Raum bereits ein eigenes interdisziplinäres Forschungsfeld etabliert hat: die Digital Game Studies (vgl. Aarseth 2006, 17f). Während die theoretischen Wurzeln der Digital Game Studies in der Narratologie und der Ludologie (vgl. Frasca 1999) zu finden sind, also Spiele entweder in ihren Textstrukturen oder in ihren Regelwerken und Simulationen in den Blick nehmen (vgl. Juul 2006), können die Forschungsaktivitäten im deutschsprachigen Raum mehrheitlich in der Tradition der Mediennutzungs- und Medienwirkungsforschung verortet werden. In den letzten Jahren ist eine zunehmende Anzahl an Arbeiten zu verzeichnen, die das Phänomen Computerspielen aus einer (medien-)pädagogischen Perspektive thematisieren (vgl. Meder/Fromme 2001, Prensky 2001, Gee 2003, Buckingham 2007, Wagner/Theunert 2007, Schorb u. a. 2008). Im Folgenden wird von der Annahme ausgegangen, dass sich ein (medien-) 
pädagogischer Zugang zu digitalen Spielen jedenfalls durch eine bildungs- und/ oder lerntheoretische Verortung auszeichnen muss.

Der hier skizzierte theoretisch-methodische Ansatz thematisiert das Phänomen Computerspiele als eine medial-kulturelle Praktik. Diese Perspektive betont die Spielhandlung, welche unhintergehbar medial gedacht werden muss, und fragt nach den Bedeutungen, welche beim Spielen produziert, reproduziert, transformiert und aktualisiert werden. Pädagogische Fragen, die sich in diesem Kontext eröffnen, fokussieren nicht die pädagogische Nützlichkeit von Computerspielen, sondern setzen an der bildungstheoretischen Bedeutung des Computerspielens an, die im Folgenden ausgehend von einem strukturalen Bildungsbegriff, wie er von Marotzki (2000) und Meder (2007) entwickelt wurde, expliziert werden soll. Nach Meder $(2007,12)$ ist Bildung als Resultat und Prozess durch ein dreifaches Verhältnis bestimmt: das Verhältnis "des Einzelnen zu den Sachen und Sachverhalten in der Welt, zu dem oder den Anderen in der Gemeinschaft (dem Sozialen), zu sich selbst in Vergangenheit, Gegenwart und Zukunft», wobei die Ausgestaltung dieser drei Verhältnisse medial begriffen wird (vgl. Swertz 2008). Werden Spiele nicht alleine als Narrationen in ihrer Spiellogik und in ihrer Wirkung begriffen, sondern als Vollzug einer spezifisch situierten medial-kulturellen Praxis, so ist im Anschluss an die bildungstheoretischen Überlegungen von Meder der bildungsrelevante Aspekt des Computerspielens angesprochen. Es eröffnet sich die Frage nach der bedeutungsstiftenden Ausgestaltung der Selbst- und Weltverhältnisse beim Spielen respektive nach der spielenden Gestaltung von Selbst- und Weltbildern.

Das Spielen ist, wie im Folgenden ausgeführt wird, auf die jeweilige Kultur bezogen, in der es hervorgebracht wird, und Spiele spiegeln gesellschaftliche Strukturen, Normen und Wertvorstellungen wider. Beim Spielen, das auf die Lebenswelt der Spielenden bezogen ist und sich gleichzeitig von ihr abgrenzt (vgl. Huizinga 1956), können Potenzialitäten realisiert und riskiert werden, da das Spiel jederzeit abgebrochen, neu gestartet, anders performiert oder beendet werden kann. Beim Spielen bieten sich dem oder der Spielenden Möglichkeiten, Ressourcen und Handlungsalternativen, die spielend aufgegriffen werden können, aber nicht müssen. Im Spiel aktualisierte Spielaktivitäten, Haltungen und Meinungen können nur innerhalb des definierten Spielrahmens Geltung beanspruchen und können so "gefahrlos» ausprobiert werden, da die (Mit-)Spielenden wissen, dass es sich «nur um ein Spiel» handelt. Spielen ermöglicht somit im Rahmen von «Als-ob»-Handlungen die Ausgestaltung von Selbst- und Weltverhältnissen in einem bildenden Sinn.

Dieser Zugang trägt dem Umstand Rechnung, dass ein Spiel auf unterschiedliche Weise gespielt werden kann und dass im Zuge dessen individuelle Bedeutungszuschreibungen seitens der einzelnen Spielenden in der Auseinandersetzung mit der sozialen und natürlichen Spiel-Welt, welche Computerspiele eröffnen und begrenzen, gestiftet werden. Mit dem hier explizierten Ansatz wird ein medien- 
pädagogischer Zugang zum Computerspielen eröffnet, der dem Phänomen des digitalen Spielens nicht normativ begegnet, sondern sich den Spielhandlungen als medial-kulturelle Praktiken fragend zuwendet und versucht, diese in ihren Bedeutungszuschreibungen zu verstehen. Vor dem Hintergrund dieser bildungstheoretischen Verortung wird der vorliegenden Arbeit in lerntheoretischer Hinsicht ein genealogischer Lernbegriff zu Grunde gelegt, der im Folgenden in Abgrenzung zu didaktisch-teleologischen Lernbegriffen, welche die aktuelle Computerspielforschung dominieren, skizziert werden soll:

Teleologische Ansätze nehmen das «Was» und «Wodurch» des Lernens in den Blick und versuchen durch eine fruchtbare Symbiose von motivationalen Aspekten des Gameplays und spezifischen Lerninhalten, das Lernen der Spielenden zu eröffnen. Exemplarisch kann dabei das Konzept des «Digital Game-Based Learnings» von Marc Prensky genannt werden (Prensky 2001), wobei digitale Spiele so designt werden, dass die Spieler durch ihr Spielerleben motiviert mit Lerninhalten in Berührung kommen und diese dadurch spielerisch angeeignet werden. Teleologisch sind diese Konzepte, weil sich der Spielvollzug dem Lernziel, nämlich dem Erwerb von Wissen, Überzeugungen und Kenntnissen unterordnet. Das Spiel wird Mittel zum Zweck des intentional vermittelten Wissenstransfers.

Kontrastierend zu diesen zielorientierten Lernkonzepten spielerischer Aneignung können genealogische Lerntheorien genannt werden (vgl. Meyer-Drawe 1982), die weniger das Ziel als den Vollzug der Spielhandlungen und der Lernprozesse in den Blick nehmen. Als genealogisch sollen hierbei jene lerntheoretischen Zugänge bezeichnet werden, die den Erfahrungsvollzug des Spielvorgangs und des Lernprozesses zum Thema haben, und nicht das Ziel der Wissensvermittlung, sondern die Genese von Erfahrungen in der kreativen Spielhandlung fokussieren (Mitgutsch 2008). Hierbei stehen weniger Spielziele und die Gestaltung von Spielregeln im Vordergrund, sondern Fragen nach der Generierung neuartiger Erfahrungsräume im Vollzug medial-kultureller Praktiken. Lernen wird dabei nicht linear, sondern zirkulär und situativ in seiner kulturellen und medialen Verflechtung verstanden. Dabei werden Momente des kritischen Reflektierens von semiotischen Domänen (Gee 2003), von neuartigen Erfahrungsräumen und -welten (Fabricatore 2000), von der Erweckung neuartiger kultureller Partizipationsmöglichkeiten (Jenkins 2007) und von mimetischen Lernvollzügen (Wulf 2007) sowie Umlernprozessen im Spielen erörtert. Je nachdem, ob das Lernen teleologisch oder genealogisch verstanden wird, kommt das Spiel in seiner Struktur und Regelung oder die medialkulturelle Handlung des Spiels und die daraus resultierenden Erfahrungen in den Blick. Dabei ergeben sich jeweils neue Möglichkeiten und Grenzen für didaktische Settings und pädagogisches Handeln. Im Kontext der vorliegenden Erörterung wird der Frage nachgegangen, wie aus einer genealogisch-lerntheoretischen und einer struktural-bildungstheoretischen Perspektive Computerspielen als medialkulturelle Praktik theoretisch gefasst und methodisch untersucht werden kann. 
Der Beitrag setzt dabei am ludic gap an, der die Differenz von Spielenden und Nicht-Mitspieler/innen markiert, indem ein medienpädagogisch-kulturtheoretischer Ansatz vorgestellt wird, mit Hilfe dessen die angesprochene Kluft zwischen Spielenden und den medienpädagogisch forschenden Nicht-Mitspieler/innen in den Blick genommen und bearbeitet werden kann. Hierzu werden theoretische und methodische Voraussetzungen entfaltet, wobei auf narratologische, ludologische und wirkungstheoretische Überlegungen Bezug genommen wird und diese Ansätze vor dem Hintergrund der explizierten bildungs- und lerntheoretischen Verortung richtungsweisend verortet werden. Dabei wird in einem ersten Schritt der grundlegenden Frage nachgegangen, welche Bedeutung das Spielen für den Menschen und sein Mensch-Sein (1) und seine kulturelle Verflechtung einnimmt (2). Im Anschluss an eine kulturtheoretische Verortung (3) erfolgt eine Beschreibung des theoretisch-methodischen Vorgehens (4), in der der Gegenstand der Analyse, die Analyseebenen und Analysekategorien vorgestellt und präzisiert werden. Am Beispiel Zoo Tycoon 2 (Blue Fang Games/Microsoft 2004) wird die digital-mediale Dimension von Computerspielen diskutiert und soll deutlich gemacht werden, inwiefern digitales Spielen als medial-kulturelle Praktik in den pädagogischen Blick kommt.

\section{Welche anthropologische Bedeutung hat das Spielen?}

Bevor digitales Spielen für pädagogische Fragestellungen zugänglich gemacht werden kann, drängt sich die grundlegende Frage nach der Bedeutung des Spiels für den Menschen auf, die in den meisten Publikationen unreflektiert bleibt. Wird diese Frage nach der Notwendigkeit des Spiels für unser Menschsein doch aufgegriffen, dann in den meisten Abhandlungen mit Verweis auf ein prominentes Zitat von Schiller: «Der Mensch spielt nur, wo er in voller Bedeutung des Worts Mensch ist, und er ist nur da ganz Mensch, wo er spielt» (Schiller 1795, 614). Im Spielen mit dem «Schönen», mit dem «Idealen» und «Zwanglosen» kommt der Mensch nach Schiller zu seiner Vervollkommnung. Nun meint dieses schillersche Spielen jedoch nicht das, was wir heute unter der Tätigkeit des Spielens verstehen. Es meint mehr und weniger zugleich. Dazu Schiller: «Freilich dürfen wir uns hier nicht an die Spiele erinnern, die in dem wirklichen Leben im Gange sind und die sich gewöhnlich nur auf sehr materielle Gegenstände richten» (Schiller 1795, 614). Schiller meint also genau nicht jene Spiele, die real und aktuell vollzogen werden, sondern das Ideal des freien Spieltriebs, das der Mensch während seines Spielens anstrebt. Das Spiel vollzieht sich bei Schiller in Gedanken und in der Fantasie, nicht an konkreten Gegenständen oder Spielzeugen. Eine Verortung des Spielens als notwendigen Bestandteil der real vollzogenen Lebenswelt des Menschens findet sich in den Schriften Eugen Finks, worin dieser das Spiel als eines der Grundphänomene neben der Arbeit, dem Kampf, der Liebe und dem Tod bezeichnet (vgl. Fink 1979). Der Mensch ist für Fink wesentlich sterblich, tätig, kämpferisch, liebend 
und spielend, wobei er dem Spiel eine spezifische Schlüsselposition zuschreibt. Im Spielen nimmt der Mensch Distanz zu den anderen Grundphänomenen und Tätigkeiten seines Lebens ein und bezieht sich umgekehrt auf diese. Während die Arbeit, der Kampf, die Liebe und der Tod direkten Zwecken folgen, wird durch das Spiel ein «zweckfreier Raum» geöffnet, der die anderen Wesenszüge des Lebens spielerisch bearbeitet, reflektiert und transformiert, ohne dabei selbst einem direkten Zweck zu folgen. Das Spiel ist für Fink der Ort des Seins, in dem der oder die Spielende erprobt, fantasiert und ziellos experimentiert - eine Oase jenseits des Zwangs und voller Freiheit.

Diese anthropologische Bedeutung des Spiels verweist auf grundlegende Bildungs- und Lernanlässe, die in medial-kulturellen Praktiken und durch sie eröffnet werden. Um in einer Gesellschaft aufzuwachsen und in eine Kultur hineinzuwachsen, benötigen Heranwachsende (und Erwachsene) Spielräume: So können sie in Distanz zur Wirklichkeit und zu ihren Zwängen treten. Gerade im Spielen wenden die Heranwachsenden (und somit der Mensch) eine Not, die durch ihre Umwelt und ihre Kultur an sie herangetragen wird. Anthropologisch kann mit Eugen Fink Spielen als Grundphänomen verortet werden, das nicht weiter ableitbar und existenziell für den Menschen ist. Der Mensch wächst im Geflecht seiner Kultur und im Einfluss seiner Mitmenschen auf und kann im Spiel die Anforderungen seiner kulturellen Entwicklung in einem zweckfreien und sanktionsfreien Raum bearbeiten. Damit ist das Verhältnis von Spiel und Kultur angesprochen. Wie kann dieses Verhältnis gedacht werden?

\section{Zum Verhältnis von Spiel und Kultur}

Ein Vertreter, der sich mit dem Verhältnis von Spiel und Kultur beschäftigt hat, ist der französische Soziologe, Literaturkritiker und Philosoph Roger Caillois. In seinem Buch Die Spiele und die Menschen weist er vielfach auf die wechselseitige Abhängigkeit von Kultur und Spiel hin und betont, dass "Spiele weitgehend von den Kulturen, in denen sie betrieben werden, abhängig sind» $(1982,91)$. Nach Caillois bieten Spiele als Spiegelbilder einer Gesellschaft dem oder der Spielenden die Möglichkeit, sich mit den jeweiligen Werten, Glaubensüberzeugungen, Rollenbildern etc. spielerisch in einem zweck- und sanktionsfreien Raum auseinanderzusetzen. Johannes Bilstein weist in diesem Zusammenhang darauf hin, dass:

[...] jede Inszenierung und Aufführung eines Spiels [...] die Aktualisierung eines kulturellen Wissens [...] ist. Indem Menschen spielen, begeben sie sich in Handlungs- und Sprachspiele hinein; dabei erfahren und modifizieren sie etwas vom kulturellen (Handlungs)wissen ihrer Gesellschaft. (Bilstein 2005, 7) 
Ein weiterer Aspekt des Zusammenhangs von Kultur und Spiel, auf den Caillois aufmerksam gemacht hat, sind die kulturübergreifenden Spielprinzipien. Anhand seiner kulturvergleichenden Beschäftigung mit Spielen zeigt er, dass Spiele zwar in Hinblick auf Bilder, Vorstellungen und Werte für jede Kultur quasi adaptiert werden, dass jedoch die Spielprinzipien (die etwa Schach und Mühle zu Grunde liegen) über die einzelne Kultur hinaus Bestand haben. Caillois beschreibt vier verschiedene Kategorien von Spielprinzipien - Agôn, Alea, Mimikry und Ilinx: "Die Neigung zum Wettkampf, das Warten auf die Chance, das Wohlgefallen an Verkleidung und die Anziehungskraft des Rausches erscheinen zweifellos als Hauptantriebe der Spiele, aber ihre Wirksamkeit durchdringt unfehlbar das ganze Leben der Gesellschaften» $(1982,95)$. Sie sind nach Caillois zähe, beständige und damit universell erscheinende Antriebe menschlicher Aktivität. Für Caillois stellt sich die Frage, welche Art von Spielen in einer Gesellschaft mit Vorliebe gespielt werden (ebd., 95)

Für unsere Auseinandersetzung mit dem Phänomen Computerspielen bedeutet dies, dass zum einen nach den gesellschaftlichen Ideologien, Werten, Bildern und Vorstellungen gefragt werden muss, die im Computerspielen zum Ausdruck kommen und Anlass zur ludischen Auseinandersetzung bieten. Zum anderen müssen die Spielprinzipien, die Spiele leiten und die als universelle Antriebe menschlicher Aktivitäten betrachtet werden können, in den Blick genommen werden. Zuletzt gilt es mit dieser Herangehensweise, lern- und bildungstheoretische Fragestellungen zu eröffnen und weiterführend zu entfalten.

Ein weiterer prominenter Vertreter, der «das Spiel als Faktor des Kulturlebens» begreift und auf dessen Überlegungen wir uns beziehen (1956/2004, 12), ist der niederländische Kulturanthropologe Johan Huizinga. In seinem viel zitierten Werk Homo Ludens geht er der Frage nach, inwiefern die «menschliche Kultur im Spiel - als Spiel - aufkommt und sich entfaltet» (ebd.). Anhand verschiedener Beispiele zeigt er, dass Kultur in ihrer ursprünglichen Phase erspielt wird (vgl. 1956/2004, 189), dass sie sich im Spiel und als Spiel entfaltet (ebd.). In diesem Zusammenhang betont er, dass Kultur nicht durch einen Entwicklungsprozess aus dem Spiel hervorgeht, sondern dass sie in Form von Spiel entsteht, «dass Kultur anfänglich gespielt wird» (Huizinga 1987, 189). Huizinga weist damit auf ein weiteres Verhältnis von Kultur und Spiel hin: Spiele können zum einen als Spiegelbilder der Gesellschaft untersucht werden, zum anderen sind es nach Huizinga die Spiele, aus denen sich Kultur entfaltet. Für unsere Beschäftigung mit Computerspielen erscheint jedoch folgender Aspekt zentral: Huizinga begreift das Spiel nicht als ein abstraktes Prinzip, sondern als aktives «Handeln» (vgl. Huizinga 1987, 16f.). Damit rückt das Spiel als Forschungsgegenstand nicht nur als kulturelles Artefakt in den Blick, sondern auch in seiner Prozesshaftigkeit bzw. als Handlung. Zu untersuchen ist laut Huizinga das Spiel auch als aktive Handlung. Damit ist eine Differenz auf- 
gemacht, die für eine medienpädagogisch-kulturtheoretische Analyse zentral ist: die Unterscheidung zwischen Spiel (Game) und Spielen (Play).

Nimmt man das Spiel in den Blick, dann zeigt sich dieses in doppelter Hinsicht: einmal als Gegenstand (Spielbrett, Spielfigur ...), der von jemandem für jemanden erzeugt wurde. Und einmal als Spielhandlung, als mediale Praktik, die eben diesem Etwas einen gewissen Sinn zuschreibt und dem Spielen eine Bedeutung verleiht. Wird die Analyse auf den Gegenstand des Spiels beschränkt, so wird die Spielhandlung, die diesem Spiel erst einen Sinn verleiht, übersehen. Untersucht man vice versa nur die Spielhandlung, liquidiert man den Gegenstand des Spielens, der wiederum die Spielhandlung wesentlich beeinflusst, ermöglicht und einschränkt. Zuletzt kann das Spiel und sein Spielen nicht von einander getrennt betrachtet werden, da es sich wechselseitig bestimmt, Sinn einräumt und Sinn ausgrenzt. Im Spielen wird Sinn gestiftet, kultureller Transfer eröffnet und es werden Bedeutungen geschaffen. Das Spiel und sein Spielen eröffnen dadurch einen Raum für lern- und bildungstheoretische Analysen kultureller Praktiken. Die Analysen des Spiels und des Spielens nehmen eine spezifische Praktik in den Blick, die uns viel über die jeweilige Kultur, die Regeln, die Riten und die Fantasien einer Gesellschaft verrät. Johannes Bilstein dazu: «Daher verschafft die Analyse spielerischer Darstellungs- und Ausdrucksformen auch einen Einblick in den kulturellen und historischen Charakter von Normen, Werten und Handlungsformen einer Gesellschaft. Auch Kinderspiele bilden hier keine Ausnahme» (Bilstein 2005, 8). Das Spiel kommt dadurch als ein Etwas in den Blick, das mehr beinhaltet als seine Regeln, sein Spielfeld und sein Spielziel. Es eröffnet in seinem genealogischen Vollzug ein Archiv kulturellen Wissens, in dem erprobt, fantasiert und ziellos experimentiert werden kann.

\section{Analyse des Computerspiel(en)s als kulturell-mediale Praktik}

Nach dieser grundlegenden Verortung des Verhältnisses von Spiel und Kultur wird im Folgenden der ludologische Ansatz des medienpädagogisch-kulturtheoretischen Zugangs zum Phänomen Computerspiel/en vorgestellt und erläutert.

\subsection{Gegenstand der Analyse}

Um den Begriff des Spielens zu bestimmen, wurde dieser von verschiedenen Autorinnen und Autoren von Merkmalen wie Arbeit und Nutzen abgegrenzt. Vielfach wurde der Begriff auch in Opposition zum Ernst gesetzt (vgl. Gebauer 1997, 1038). In dem Werk Wahrheit und Methode (1990) zeigt Gadamer im Kontext der geisteswissenschaftlichen Hermeneutik die Problematik dieser Begriffsstrategie auf, indem er darauf hinweist, dass z. B. der Ernst eine enorme Bedeutung für das Spiel hat, "dass Spielen einen eigenen Wesenszug des Ernsten hat. [...] Wer das Spiel nicht ernst nimmt, ist ein Spielverderber. [...] nur der Ernst beim Spiel lässt das Spiel ganz Spiel sein» (Gadamer 1990, 108). Der Gegenbegriff des Spiels ist 
für Gadamer daher nicht der Ernst, sondern die negierte Spielhandlung, nämlich das Nicht-Dabeisein beim Spielen, das Nicht-Drinnensein in der Spielwelt und das Nicht-Mehr-Mitspielen. An dieser Stelle setzt unser Verständnis von Spielen an: Was als Spielen in den Blick kommt, wird dadurch entschieden, ob die jeweilige Person einen ludischen Sinn in ihre jeweilige Tätigkeit legt. Das Spiel wird erst durch die Sinnzuschreibung des oder der Spielenden zum Spiel.

Auf diesen Aspekt verweist auch Gregory Batson, der sich mit dem Phänomen Spiel(en) aus einer kommunikationstheoretischen Perspektive auseinandersetzt. Er unterscheidet in seiner Theorie des Spiels und der Phantasie (1985) zwischen dem Spielrahmen und der Spielhandlung. Der Spielrahmen bezeichnet in seinen Überlegungen einen metakommunikativen Rahmen, welcher als gedachte, kommunikative Begrenzung Spielhandlungen aus der Alltagswelt herauslöst (Gebauer 1997, 1041). So ist nach Batson ein konstitutives Merkmal von Spielen, dass «die beteiligten Organismen in gewissem Masse der Metakommunikation fähig sind, d.h. Signale austauschen können, mit denen die Mitteilung «Dies ist ein Spiels übertragen wird» (Batson 1985, 244). D. h., die Spielenden müssen, damit ein Spiel stattfinden kann, zwischen Spiel und Nichtspiel differenzieren können. Gibt es keine (explizite oder implizite) metakommunikative Verständigung darüber, dass jetzt gespielt wird, kann man nach Batson nicht vom Phänomen Spielen sprechen. In diesem Sinne wird der Forschungsgegenstand über die spielenden Personen erschlossen, die uns über ihre Spieltätigkeit und den Spielsinn im Rahmen von Befragungen Auskunft geben.

\subsection{Analyseebenen}

Im Anschluss an den Pädagogen Hans Scheuerl performiert sich die ludische Praktik in drei spezifischen Dimensionen (vgl. Scheuerl 1981, 47): (1) In Form von Spielen als innovative oder tradierte Vereinbarungs- und Regelgebilde, (2) als Spieltätigkeit, in der Spiele nach den Strukturen praktiziert, variiert und performiert werden, und (3) im Spielen als Bewegung, welches sich durch die Momente Freiheit, Ambivalenz, relative Geschlossenheit sowie die räumliche und zeitliche Begrenzung auszeichnet. Diese drei Dimensionen des Spielens sollen in weiterer Folge als (1) Game (als geregeltes und gerahmtes Spiel), (2) Gameplay (als das sinnstiftende Bewegen und das Tun im Spiel) und als (3) Play (als das unkontrollierte, anarchische und kreative Moment des Spielens) bezeichnet werden (vgl. dazu Caillois 1982/1958; Frasca 1999; Adamowsky 2005). Das Game impliziert die Regeln, das Spielfeld, die Spielfiguren und die Grenzen des Spiels, was im Kontext digitaler Spiele auch die technologische Umsetzung auf das Medium Computer umfasst. Die mediale Gestaltung der Regeln, des Orts, der Grenzen und der Spielfiguren bestimmt hierbei in besonderer Weise, welches Gameplay und welche Momente des Plays bedient werden können. Hierbei kann auch bestimmt werden, welche Inhalte den Spielenden zur Verfügung stehen, mit welchen Metaphern im 
Spiel gearbeitet wird und welche Story dem Spiel zu Grunde gelegt wird. In den meisten Analysen digitaler Spiele wird allein auf die Dimension des Games fokussiert. Anhand von teilnehmenden Beobachtungen (z. B. durch thinking aloud) in Kombination mit qualitativen Interviews kann jedoch die Play-Dimension, die sich durch Ambiguität, Spontanität und Flexibilität auszeichnet, erfasst werden. Das Game, das als eine institutionalisierte Struktur charakterisiert wird, in der sich das Play entfalten kann, wird anhand von Spielbeschreibungen seitens der Hersteller und Spielenden gefasst. Game und Play voneinander zu unterscheiden bedeutet, zwischen der Struktur von Spielen und der unmittelbaren Spielhandlung zu differenzieren. Games regeln das Play und eröffnen bestimmte Spielhandlungen, sie ermöglichen und verhindern ein bestimmtes Tun und verweisen damit auf die Grenzen und Beschränkungen bzw. Erfordernisse und Möglichkeiten des spezifischen Spielgeschehens.

\subsection{Analysekategorien}

Die Analyse von Play und Game erfolgt vor dem Hintergrund von fünf Analysekategorien, die in Anlehnung an Caillois entwickelt wurden. Caillois unterscheidet, wie bereits angesprochen, zwischen den vier Spielkategorien Agôn (Wettkampf), Alea (Glücksspiel), Mimikry (Verkleidung/Schauspiel) und Ilinx (Rausch/Trance). Spiele können in Hinblick auf diese Prinzipien durch Analysen unterschieden werden. Die Analyse nach Spielkategorien kann nicht nur auf traditionelle Spiele angewandt werden, sondern auch auf Computer-, Video- und Konsolenspiele. Hierzu scheint jedoch ein gewisses «mediales Update» vonnöten, damit die Spieltheorie Caillois' die medialen Implikationen der digitalen Spiele des 21. Jahrhunderts zu fassen vermag. Aus diesem Grund empfiehlt Adamowsky (2005) eine Erweiterung dieser vier Elementarkategorien des Spiels (Agôn, Alea, Mimikry und llinx) um eine fünfte Kategorie - das Experiment (vgl. Adamowsky 2005). Das spielerische Experimentieren gehört weder zum Wettkampf, noch zum Glücksspiel, noch zur Nachahmung oder zum Rausch, sondern zeigt sich allein in der Freude an der Weiterentwicklung und der Modellierung (siehe Tabelle 1).

Eine zweite Erweiterung dieser nun fünf Kategorien des Spielens betrifft die Elementarkategorie des Rausches (llinx) im Spiel. Denn der durch körperliche Betätigung hervorgerufene Rauschzustand (z. B. durch Drehung) ist bei üblichen Spielkonsolen schwer zu erlangen ${ }^{1}$. Und doch werden beim Computerspielen vielfach rauschähnliche Zustände beobachtet, die man als Flow (Csikszentmihalyi 1997; Fritz 2002) bezeichnen könnte. Durch die - den Spielstärken des oder der Spielenden entsprechenden - Balance zwischen Können und Anforderung verschmilzt die Aufmerksamkeit des Spielers oder der Spielerin mit den Strukturen des Spiels: «Die Spieler verschmelzen mit dem Spiel, sie gehen auf in der Spieltätigkeit (〈Flow〉), ha-

Die Ende letzten Jahres 2007 auf dem Markt erschienene Wii von Nintendo stellt hierbei eine Ausnahme dar. 
ben Schwierigkeiten, aufzuhören und ‘vergessen` die Zeit.» (Fritz/Misek-Schneider 1995). ${ }^{2}$ Im Kontext unserer Analyse von digitalen Spielen wird in weiterer Folge vom Flow als Elementarkategorie neben Agôn, Alea, Mimikry und Experiment ausgegangen [Tabelle 1]:

\begin{tabular}{|c|c|c|c|c|c|}
\hline & $\begin{array}{l}\text { AGON } \\
\text { [Wettkampf] }\end{array}$ & $\begin{array}{l}\text { ALEA } \\
\text { [Chance] }\end{array}$ & $\begin{array}{l}\text { MIMICRY } \\
\text { [Verkleidung] }\end{array}$ & $\begin{array}{l}\text { ILINX / Flow } \\
\text { [Rausch] }\end{array}$ & $\begin{array}{l}\text { EXPERIMENT } \\
\text { [Modellieren] }\end{array}$ \\
\hline PLAY [paidia] & $\begin{array}{l}\text { Nichtgeregel- } \\
\text { ter Wettlauf, } \\
\text { Kampf, } \\
\text { Athletik }\end{array}$ & $\begin{array}{l}\text { Auszählspiele, } \\
\text { Kopf oder Zahl }\end{array}$ & $\begin{array}{l}\text { Kindliche } \\
\text { Nachahmung, } \\
\text { Illusionsspiele, } \\
\text { Puppe, Maske }\end{array}$ & $\begin{array}{l}\text { Kindliche } \\
\text { Drehspiele, } \\
\text { Zirkus,Schaukel, } \\
\text { Walzer, Flow }\end{array}$ & Bausteine \\
\hline \multicolumn{6}{|c|}{ GAMEPLAY [sinnstiftendes Bewegen und Tun im Spiel] } \\
\hline $\begin{array}{l}\text { GAME [ludus] } \\
\text { Kreuzwort- } \\
\text { rätsel }\end{array}$ & $\begin{array}{l}\text { Sportwett- } \\
\text { kämpfe im All- } \\
\text { gemeinen }\end{array}$ & $\begin{array}{l}\text { Einfache Lotte- } \\
\text { rie, Lotterie auf } \\
\text { Buchung }\end{array}$ & $\begin{array}{l}\text { Schaukünste im } \\
\text { Allgemeinen }\end{array}$ & $\begin{array}{l}\text { Alpinismus, } \\
\text { Kunst-Sprünge }\end{array}$ & $\begin{array}{l}\text { Chemie-Bau- } \\
\text { kasten, Strate- } \\
\text { giespiele }\end{array}$ \\
\hline
\end{tabular}

Wie kann diese ludologische Perspektive auf Spiele und jene Spielhandlungen, die sich innerhalb der Spielstrukturen performieren, als mediale und kulturelle Praktik gedacht werden?

\section{Computerspielen als medial-kulturelle Praktik}

Spielen war - auch vor der Entwicklung und Etablierung massenmedialer Technologien - eine mediale Praktik. Die Spielenden nutzen notwendigerweise Medien - die Sprache (vgl. Gadamer 1990), den Leib/Körper, materielle Artefakte etc. -, um in eine Spielhandlung zu treten und dieser Sinn zu verleihen sowie um mit den Mitspielenden zu kommunizieren. Im Fall von digitalen Spielen kommen hierbei digitale Medien zum Einsatz, die spezifische Spielräume mit eigentümlichen Qualitäten, Möglichkeiten und Grenzen erzeugen, welche Spielen als medial-kulturelle Praktiken mitbedingen. Im Folgenden soll beispielhaft am Spiel Zoo Tycoon 2 (Blue Fang Games/Microsoft 2004) verdeutlicht werden, welche Möglichkeiten und Grenzen durch die technisch-mediale Vermittlung des Spiels und seinem Spielen eröffnet werden.

Beim Spiel Zoo Tycoon 2 handelt es sich in erster Linie um ein Wirtschaftssimulationsspiel, wobei das seitens der Entwicklerinnen und Entwickler definierte Spielziel darin besteht, einen Zoo zu entwerfen, diesen aufzubauen, mit unterschiedlichen

2 Fritz und Misek-Schneider unterscheiden hierbei noch zwischen der Frustrationsspirale (flow durch den Wunsch der Überwindung) und der positiv-emotionalen Spielfolge (Wiederherstellung der «Lust»). Darauf kann hier nicht weiter eingegangen werden (vgl. dazu http://snp.bpb.de/snp/referate/fritzfzt.htm [1.11.2008]). 
Tieren und Gehegen auszustatten und dabei einen möglichst grossen Profit bei einer hohen Besucherzufriedenheit zu erwirtschaften. Um den spezifischen Anforderungen der Pflege und der artgerechten Haltung der Tiere zu entsprechen, beinhaltet das Spiel ein Zoo Lexikon, in dem Teile der Microsoft-Enzyklopädie Encarta zu finden sind, die die Spielenden zur Lösung der Anforderungen benötigen. Ohne an dieser Stelle dem Aspekt nachgehen zu können, welche komplexen und umfassenden Wissensinhalte sich Jugendliche im Rahmen dieses Spiels problemund handlungsorientiert aneignen, soll der Frage nachgegangen werden, welche medial-kulturelle Praktik das Spiel im technischen Bereich ermöglicht.

Ein besonderes Feature im Spiel Zoo Tycoon 2 stellt die Möglichkeit dar, im Spiel aus zwei unterschiedlichen Perspektiven zu agieren. Während man sich als Managerin oder Manager um die Gestaltung und das Management des Zoos kümmert, wird der Zoo aus einer Vogelperspektive gezeigt (siehe Abbildung 1). Aus dieser Perspektive kümmert man sich um die strategische Planung und Organisation des Zoos. Zugleich kann jede/r beliebige Besucher/in oder Mitarbeiter/in des Zoos via Mausklick aktiviert und auf dessen/deren First-Person-View gewechselt werden (siehe Abbildung 2) und somit der Zoo aus dieser alternativen Perspektive erlebt werden. Durch die Einführung dieser Perspektive versuchten die Entwicklerinnen und Entwickler dem Wunsch der Spielenden nachzukommen, den Tieren «näher» zu kommen und direkt mit ihnen zu interagieren. Die Spielenden können dabei die Tiere «berühren», «streicheln» und «pflegen» - was das Wohlbefinden der Tiere steigert.

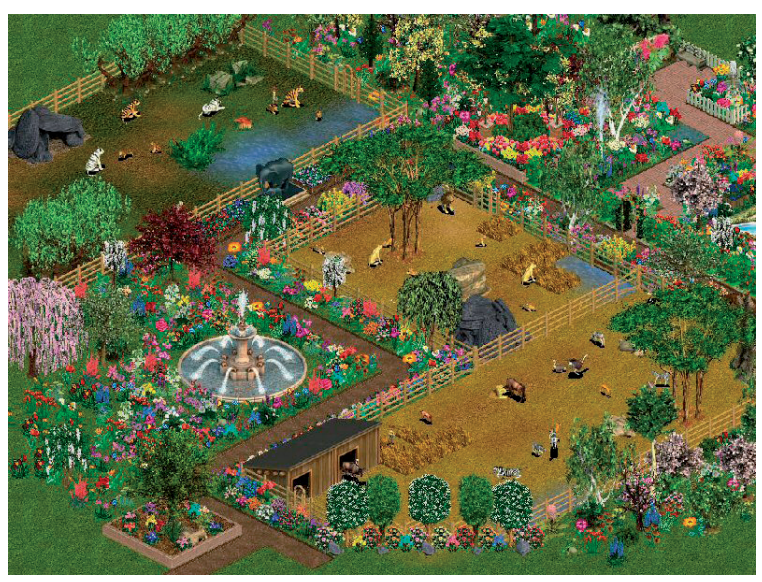

Abbildung 1

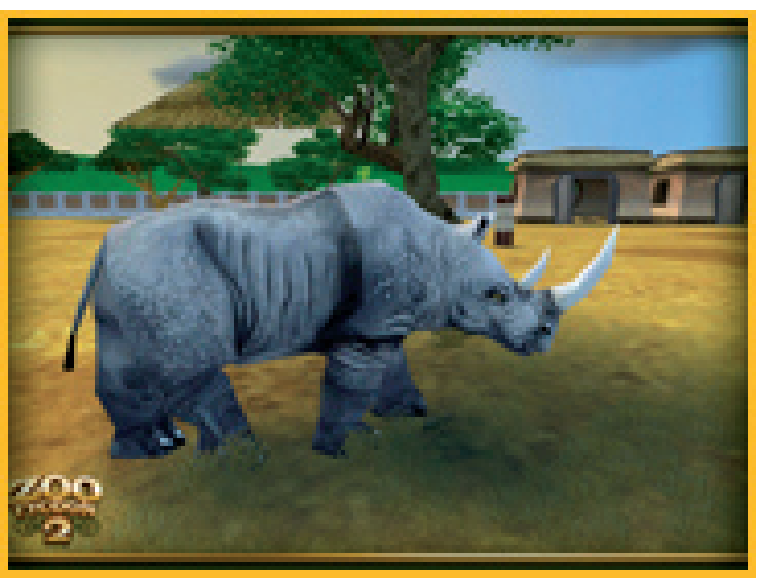

Abbildung 2

Die Implementierung dieser zweiten First-Person-Perspektive veränderte das Gameplay von Zoo Tycoon 2 im Vergleich zu seinem Vorgänger (Blue Fang Games/ Microsoft 2001) in besonderer Art und Weise. Es eröffnet dem Spiel eine zweite 
Spielweise, denn zum einen können die Besucherinnen und Besucher Fotos der Tiere schiessen und zum anderen ist es nun möglich, mit den Tieren in der Rolle eines Pflegers direkt in Kontakt zu treten. Für die Spielenden bedeutet dies, dass sie auf unterschiedlichen Ebenen agieren können, durch die ein doppeltes Erleben des Zoos zugänglich wird. Sie können den Zoo zunächst planen und errichten und dann als Besucherin oder Besucher diesen selbst durchwandern und betrachten. Dieses Feature eröffnet unterschiedliche Zugänge zum Spiel und erlaubt eine Kombination aus unterschiedlichen Spielhandlungen: Unter Bezugnahme auf die oben erläuterten Analysekategorien (4.3) kann der Zoo geplant und entworfen [Kat. Modellieren], in der Rolle des Wärters oder der Wärterin erlebt [Kat. Verkleidung] oder aber auch (in den vielen integrierten Geschicklichkeitsspielen) als Herausforderung gesehen werden [Kat. Wettkampf]. Die Kombination aus Wirtschaftsstrategie (Manager-Perspektive) und dem Agieren als Pflegerin oder Pfleger (First-Person-Perspektive) eröffnet wesentliche spielerische (Play) Aspekte im Gameplay und transformiert dadurch eine spezifische medial-kulturelle Praktik.

Die medial-kulturelle Praktik des Computerspiels Zoo Tycoon 2 spiegelt sich in unterschiedlichen medialen Artefakten (Foren, Videos, Screenshots etc.) wider. Besonders die Möglichkeit in der 3-D-First-Person-Perspektive beim Zoobesuch die Tiere zu fotografieren, erscheint dabei ausschlaggebend. Mittlerweile existieren verschiedene Online-Communitys zum Spiel Zoo Tycoon 2, in denen besonders gute, originelle und witzige Fotos der Spielenden der Öffentlichkeit präsentiert werden. ${ }^{3}$ Aber auch über das Videoportal YouTube ${ }^{4}$ werden unterschiedliche Filme vom eigenen Zoo veröffentlicht und ausgetauscht. Hierbei zeigt sich eine besondere Form der Medienkonvergenz (vgl. Wagner/Theunert 2007), die vom Gamedesign zwar angelegt wurde, aber erst durch die Spielenden hergestellt werden kann. D. h., die Spielenden haben durch ihr Play das Game Zoo Tycoon 2, welches von der Struktur nur im Einzelspielmodus gespielt werden kann, in ein «OnlineMehrspielermodus-Spiel» transformiert, indem sie über das Medium Internet Erfahrungen in Bezug auf Gestaltung des Zoos, Pflege der Tiere etc. austauschen. Kommt in diesem Sinn digitales Spielen als Form medial-kultureller Praktik in den Blick, können Spiele und Spielhandlungen in ihrem jeweiligen sozialen Setting aus unterschiedlichen Ebenen (Game, Play, Gameplay) und nach den bestimmenden Kategorien unter Berücksichtigung der technisch-medialen Transformation analysiert werden. Spielen kann dabei nicht unabhängig von dem «situativen und atmosphärischen Kontext, dem Handlungs-, Erfahrungs- und Erlebnisgefüge» (Matussek u. a. 2000, 201), in dem beispielsweise mit dem artifiziellen Medium Computer umgegangen wird, gedacht werden. Im Spielen erleben, experimentieren, erproben und erlernen die Spielenden neue Gebrauchsweisen und Gestaltungsformen, die sich «nicht in der Bedienung von Apparaten» (ebd.) erschöpfen. Die Medien-

3 Siehe http://zootycoon.com/community/fansites.htm [1.11.2008]

4 Siehe http://www.youtube.com/ [1.11.2008] 
pädagogik kann besonders in diesem Forschungsfeld bedeutsame Erkenntnisse und Einsichten einbringen, die nicht nur den digital divide, sondern auch den ludic gap zwischen Spielenden und Nicht-Spielenden erkennen lassen und einen konstrukiven Umgang mit diesem ermöglichen.

Beim Computerspielen als medial-kulturelle Praktik in Relation zu Spielregeln und Spielvollzug kann die Transformation und Umkehrung von Erfahrungsvollzügen im Lernprozess genealogisch untersucht werden. Dabei wird deutlich, welche neuartigen Erfahrungsräume im Spiel eröffnet werden und inwiefern dadurch ein Um-, Dazu- und Erlernen als situiert-kulturelle Handlung vollzogen wird. Lernen aus digitalen Spielen als kulturell-mediale Praktik bedeutet im Spielen die eigenen Erwartungen zu konfrontieren und im sanktionsfreien Raum die bisherigen Erfahrungen an ihre Grenzen zu führen (vgl. Mitgutsch 2008). Dabei wird in strukturalbildungstheoretischer Hinsicht das Zusammenspiel von Selbst- und Weltbild fokussiert, das beim digitalen Spielen auf die Probe gestellt wird. Bildung meint hier, das Selbst beim Spielen in Relation zur Welt aufs Spiel zu setzen und die eigentlichen Möglichkeitsräume spielerisch auszuloten. Diese lern- und bildungstheoretische Perspektive auf die medial-kulturelle Praktik des digitalen Spielens nimmt die/den Spielende/n in ihrer/seiner medialen, situierten, kulturellen und sozialen Verwobenheit in den Blick und fragt nach individuell bedeutungsvollen Spielhandlungen. In diesem Beitrag wird im Sinne eines theoretisch-konzeptionellen Entwurfs ein möglicher Zugang skizziert, der medienpädagogische Analysen an konkreten Spielen vor dem Hintergrund der hier skizzierten Lern- und Bildungstheorien ermöglicht - ein Forschungsdesiderat, dessen sich die Medienpädagogik anzunehmen hat. 


\section{Literatur}

Aarseth, Espen (2006). Warum Game Studies. In: Kaminski, Winfrid/ Lorber, Martin (Hrsg.) Computerspiele und soziale Wirklichkeit. München: kopaed. 25-33.

Adamowsky, Natascha (2005). Spielen und Erkennen - Spiele als Archive. In: Anthropologie und Pädagogik des Spiels. Weinheim und Basel: Beltz. 37-52.

Batson, Gregory (1985). Ökologie des Geistes. Anthropologische, psychologische, biologische und epistemologische Perspektiven. Frankfurt/Main: Suhrkamp.

Bilstein, Johannes (Hrsg.) (2005). Anthropologie und Pädagogik des Spiels. Weinheim und Basel: Beltz.

Buckingham, David (2007). Beyond Technology: Children's Learning in the Age of Digital Media. Cambridge: Polity Press.

Caillois, Roger (1982/1958). Die Spiele und die Menschen: Maske und Rausch. Frankfurt/M: Ullstein.

Csikszentmihalyi, Mihaly (1997). Finding Flow: The psychology of engagement with everyday life. New York: Harper Collins.

Fabricatore, Carlo (2000). Learning and Videogames: An Unexploited Synergy. Paper presented at AECT 2000. http://www.learndev.org/dl/FabricatoreAECT2000.pdf [1.11.2008]

Fink, Eugen (1979). Grundphänomene des menschlichen Daseins. Freiburg: Verlag Rombach.

Foucault, Michel (1981/1969). Die Archäologie des Wissens. Frankfurt: Suhrkamp.

Frasca, Gonzalo (1999). Ludology meets narratology: Similitude and differences between (video)games and narrative. Finnish as Ludologia kohtaa narratologian in Parnasso, 3: 1999. http://www.ludology.org/articles/ludology.htm [1.11.2008].

Fritz, Jürgen (2003). Zwischen Frust und Flow: Vielfältige Emotionen begleiten das Spielen am Computer. In: Fritz, Jürgen / Fehr, Wolfgang (Hrsg): Computerspiele. Virtuelle Spiel- und Lernwelten. Bonn 2003 (CD-Rom).

Fritz, Jürgen / Misek-Schneider, Karla (1995). Computerspiele aus der Perspektive von Kindern und Jugendlichen. In: Computer + Unterricht 19: 45-47.

Fromme, Johannes (2007). Spiele in virtuellen Umgebungen. Überlegungen zur Beschreibung und Analyse eines neuen Mediums. In: Hartwich Dietmar./ Swertz, Christian / Witsch, Monika (Hrsg.): Mit Spieler. Überlegungen zu nachmodernen Sprachspielen in der Pädagogik, Würzburg: Königshausen \& Neumann. 9-29.

Gadamer, Hans-Georg (1990) [1960]. Wahrheit und Methode. Grundzüge einer philosophischen Hermeneutik. Band 1. Tübingen: J.C.B. Mohr.

Gee, James Paul (2003). What Video Games Have to Teach Us About Learning and Literacy. New York: Palgrave/ Macmillan.

Gebauer, Gunter (1997) Spiel. In: Wulf, Christoph (Hrsg.) Handbuch Historische Anthropologie, Weinheim und Basel: Beltz. 
Huizinga, Johan (1956/1938). Homo ludens: Vom Ursprung der Kultur im Spiel. Reinbek bei Hamburg: Rowohlt.

Jenkins, Henry (2006). Convergence Culture. Where Old and New Media Collide. New York: University Press.

Juul, Jasper (2006). Half-Real: Video Games between Real Rules and Fictional Worlds. Cambridge, Massachusetts: MIT Press.

Matussek, Peter (2000). Mediale Praktiken. In: Böhme, Hartmut/ Matussek, Peter/ Müller, Lothar (Hrsg.): Orientierung Kulturwissenschaft: was sie kann, was sie will, Reinbek bei Hamburg: Rowohlt.

Meder, Norbert / Fromme, Johannes (Hrsg.) (2001). Bildung und Computerspiele: zum kreativen Umgang mit elektronischen Bildschirmspielen, Opladen: Leske und Budrich.

Meyer-Drawe, Käte (1982). Lernen als Umlernen. Zur Negativität des Lernprozesses. In: Meyer-Drawe, Käte; Lippitz, Wilfried (Hrsg.) (2008). Lernen und seine Horizonte: Phänomenologische Konzeptionen menschlichen Lernens - didaktische Konsequenzen. Königstein, 19-45.

Mitgutsch, Konstantin (2008). Digital Play.Based Learning. A philosophical-pedagogical perspective on learning and playing in computergames. In: Special issue of the Journal for Information Technology Studies as Human Science. Human IT (9.3). Online: http://etjanst.hb.se/bhs/ith/3-9/index.htm

Schorb, Bernd / Kiessling, Matthias / Würfe, Maren / Keilhauer, Jan (2008). Medienkonvergenz Monitoring Online-Spieler-Report 2008. Die Online-Spieler: Gemeinsam statt einsam. http://www.uni-leipzig.de/ umfmed/MeMo_OSR08. pdf [1.11.2008]

Schrammel, Sabrina / Mitgutsch, Konstantin (2008). Spielerische Gewalt. Die ludische Kultur des Computerspielgenres «Shooter». In: Mitgutsch, Konstantin / Rosenstingl, Herbert (Hrsg.). Faszination Computerspielen: Theorie - Kultur - Erleben. Wien: Braumüller Verlag, 69-83.

Prange, Klaus / Strobel-Eisele, Gabriele (2006). Die Formen des pädagogischen Handelns. Eine Einführung, Stuttgart: Kohlhammer.

Prensky, Marc (2001). Digital Game-Based Learning. New York: McGraw-Hill.

Schiller, Friedrich (1795). Über die ästhetische Erziehung des Menschen; Online unter: http://gutenberg.spiegel.de/?id=5\&xid=2407\&kapitel=1 [1.11.2008].

Scheuerl, Hans (1981). Zur Begriffsbestimmung von «Spiel» und «spielen». In: Röhrs, Hermann (Hrsg.), Das Spiel, ein Urphänomen des Lebens. Wiesbaden: Akademischer Verlagsgesellschaft. 41-51.

Schrammel, Sabrina (2008). Play based learning. Die Aktivität des Computerspielens als Lernanlass. In: Mitgutsch, Konstantin / Rosensingl, Herbert (Hrsg.). Faszination Computerspielen. Theorie - Kultur - Erleben. Wien: Braumüller Verlag. 69-83. 
Sesink, Werner (2007). Bildung und Medium. Bildungstheoretische Spurensuche auf dem Felde der Medienpädagogik. In: Jahrbuch Medienpädagogik 6: Medienpädagogik - Standortbestimmung einer erziehungswissenschaftlichen Disziplin. Hrsg. v. Werner Sesink, Michael Kerres u. Heinz Moser. Wiesbaden: VS-Verlag, 2006. 70-100.

Swertz, Christian (2008). Der Bildungsbeitrag des Digital Play-Based Learnings. In: Mitgutsch, Konstantin / Rosenstingl, Herbert (Hrsg.). Faszination Computerspielen. Theorie - Kultur - Erleben. Wien: Braumüller Verlag. 127-137.

Wagner, Ulrike / Theunert, Helga (2007). Konvergenzbezogene Medienaneignung in Kindheit und Jugend. In: MedienPädagogik 14 (11.12.2007): http://www.medienpaed.com/14/wagner_theunert0712.pdf [1.11.2008].

Wulf, Christoph (2007). Lernkulturen im Umbruch. Rituelle Praktiken in Schule, Medien, Familie und Jugend. Wiesbaden: VS Verlag.

\section{Abbildungen und Tabellen}

Abbildung 1: http://members.surfbest.net/klb123@surfbest.net/Zoo008.jpg Abbildung 2: http://zootycoon.com/

Tabelle 1: Verteilung der Spiele nach Roger Caillois 1982/1958 \& Adamowsky 2005 